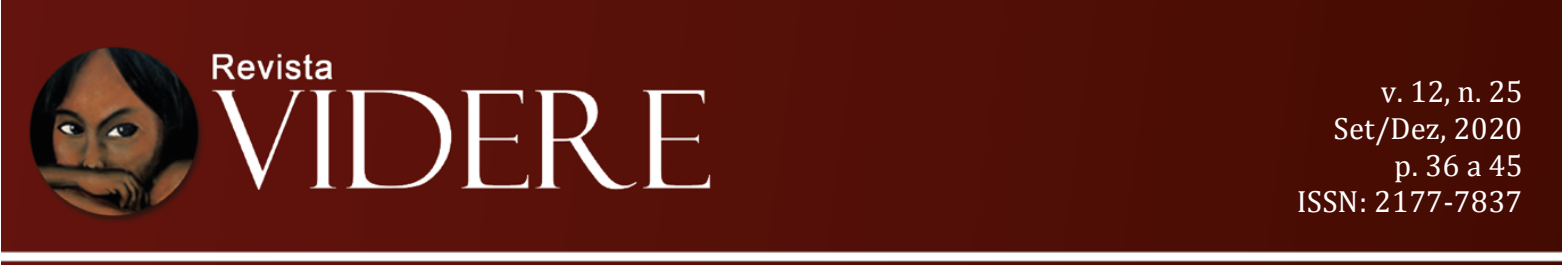

\title{
PROTEZIONE DEI DIRITTI UMANI IN OCCASIONE DI EVENTI NATURALI SECONDO IL COMITATO ONU IN TEITIOTA C. NUOVA ZELANDA: DALLA TEORIA DEGLI OBBLIGHI POSITIVI DI TUTELA AL DIVIETO DI RESPINGIMENTO.
}

\author{
THE UN COMMITTEE TEITIOTA V NEW ZELAND'S DECISION AND THE HUMAN \\ RIGHTS' PROTECTION IN THE CONTEXT OF NATURAL EVENTS: FROM THE \\ THEORY OF POSITIVE OBLIGATIONS TO THE PROHIBITION OF REFOULEMENT
}

\begin{abstract}
LA PROTECCIÓN DE LOS DERECHOS HUMANOS FRENTE A LOS DESASTRES Y CAMBIO CLIMÁTICO, SEGÚN EL COMITÉ DE LA ONU EN TEITIOTA C. NUEVA ZELANDA : DESDE LA TEORÍA DE LAS OBLIGACIONES POSITIVAS DEL ESTADO HACIA EL PRINCIPIO DE NO DEVOLUCIÓN.
\end{abstract}

Michela Castiglione

Dottore di ricerca in Scienze Giuridiche

Università di Pisa (Italia)

E-mail: michecastiglione@gmail.com OrcidID: https://orcid.org/0000-0002-5594-9841

\begin{abstract}
In its Teitiota v. New Zeland's landmark decision, The UN Human Rights Committee set out two fundamental principles of international law. It recognizes that the effects of climate change and natural disasters may give rise to violations of the right to life and the prohibition of inhuman or degrading treatment, and thus trigger the non-refoulment obligations of sending states. The purpose of this paper is to consider how The UN Human Rights Committee Teitiota's decision may facilitate the protection of environmentally displaced persons under the 1984 Cartagena declaration.
\end{abstract}

Keywords: climate change. forced migration. positive obligations to protect. principle of nonrefoulement. Cartagena declaration on refugees.

Abstract: con la decisione del 7 gennaio 2020 resa in Teitiota c. Nuova Zelanda resa in Teitiota c. Nuova Zelanda, Il Comitato dei diritti umani dell'Onu riconosce due fondamentali principi di diritto internazionale: da un lato ammette che gli effetti dei cambiamenti climatici possano comportare una violazione del diritto alla vita e del divieto di trattamenti inumani o degradanti e, dall'altro, per la prima volta in assoluto, stabilisce che il rischio di subire tali effetti nel Paese di origine genera il divieto di respingimento da parte di Stati terzi. Con questo contributo si andrà ad analizzare come questo precedente possa rappresentare uno 
spunto di riflessione interpretativo per avanzare istanze di tutela di protezione ai sensi della dichiarazione di Cartagena sui rifugiati del 1984.

Keywords: cambiamento climático. obblighi positivi di tutela. migrazione forzata. divieto di refoulement. Dichiarazione di Cartagena.

Resumen: La decisión del 7 de enero de 2020 del Comité de Derechos Humanos de la ONU sobre el caso de Teitiota v. en Nueva Zelanda se destaca por reconocer dos fundamentales principios del derecho internacional: por un lado establece que los efectos del cambio climático pueden implicar una violación del derecho a la vida y a la prohibición de tratos inhumanos o degradante y, ademas, por primera vez, establece que el riesgo de sufrir tales efectos en el país de origen genera la prohibición de devolución por parte de terceros países. En el presente artículo se irá analizando cómo esta decisión puede representar pistas de reflexión interpretativa para brindar protección bajo la Declaración de Cartagena sobre Refugiados de 1984.

Palavras-chave: cambio climático. obligaciones positivas del Estado. migración forzada. principio de devolución. Declaración de Cartagena.

\section{Introduzione}

Negli ultimi decenni eventi meteorologici avversi e fenomeni di degradazione ambientale stanno aumentando in frequenza e intensità. Il cambiamento climatico è visto come la principale causa di fenomeni ambientali e naturali come siccità, innalzamento del livello del mare, desertificazione, cicloni e alluvioni. I geologi definiscono questa situazione Antropocene cioè «l'epoca geologica in cui l'ambiente terrestre, inteso come l'insieme delle caratteristiche chimiche e biologiche in cui si svolge ed evolve la vita, è fortemente condizionato a scala sia locale sia globale dagli effetti dell'azione umana» (TRECCANI, 2012). Secondo gli ultimi dati dell'Internal Displacement Monitoring Centre i disastri rappresentato la principale causa di sfollamento interno di persone al mondo. Nel 2019 eventi improvvisi e a lenta insorgenza hanno causato 23,9 milioni di sfollati, registrando un aumento rispetto all'anno precedente di 6,7 milioni; contro i 8, 5 milioni di sfollati indotti da guerre e conflitti (2020, p. 9) L'America latina è particolarmente esposta a questi eventi e il Brasile è uno dei maggior stati della regione ad esserne colpito. Nel 2019 si sono verificati più di 500 disastri, per la maggioranza inondazioni e frane ma anche eventi a lenta insorgenza come siccità, erosione costiera che hanno provocato 295 mila sfollamenti interni (2020, p. 57). Pur in assenza di dati ufficiali, è riconosciuto ampiamente che il cambiamento climatico e l'impatto degli eventi naturali possano indurre le persone a migrare forzatamente anche oltre i 
confini del proprio Stato (UNHCR, 2019, p. 12). Lo stretto legame esistente tra protezione dell'ambiente e realizzazione di una vasta gamma di diritti umani è oramai riconosciuto a livello internazionale. Nel 2015 il Consiglio dei diritti umani delle Nazione Unite ha commissionato un ampio progetto di ricerca, Mapping Human Rights Obligations Relating to the Enjoyment of a Safe, Clean, Healthy and Sustainable Environment, che ci ha restituito una encomiabile panoramica dell'evoluzione giurisprudenziale in tema di collegamento tra eventi naturali e ambientali e obblighi positivi di tutela incombenti sugli Stati, resa dai vari Comitati e Corti deputati al controllo sull'attuazione dei rispettivi strumenti di diritto internazionale e regionali a presidio della tutela dei diritti umani. Il Global Compact on Refugees (UNITED NATIONS, 2018, p. 11) evidenzia la necessità di garantire protezione alle persone che si trovano oltre i confini del proprio Stato in occasione e/o per l'effetto del cambiamento climatico e di eventi naturali, attraverso l'impiego di meccanismi di protezione complementari regolati anche dagli ordinamenti domestici. L'Assemblea Generale delle Nazioni Unite, con la bozza di risoluzione Providing legal protection for persons displaced by the impacts of climate change (2019, p. 4) decide di sviluppare uno strumento internazionale legalmente vincolante al fine di creare una protezione appropriata per le persone sfollate a causa degli impatti del cambiamento climatico.

\section{Fatti di causa e le risultanze del comitato ONU}

É alla luce di quanto brevemente premesso che deve essere letta la nota decisione del 7 gennaio 2020 del Comitato dei diritti umani dell'Onu (di seguito Comitato Onu) resa in Teitiota c. Nuova Zelanda.

Prima di procedere, pare utile delineare brevemente i fatti di causa e le risultanze in diritto. Il caso trae origine dal Sig. Teitiota, cittadino della Repubblica del Kiribati, un atollo dell'Oceano pacifico del Sud, che si era visto negato dalla Nuova Zelanda il riconoscimento dello status di rifugiato ai sensi della Convenzione di Ginevra e che pertanto era stato rimpatriato nel Paese di origine. Il ricorrente, reiterando le doglianze già esposte dinnanzi alle Corti neozelandesi, adiva così il Comitato ONU ritenendo che il ritorno nel proprio Paese di origine lo avesse esposto, insieme alla sua famiglia, a un serio rischio per la vita in violazione dell'art. 6 del Patto ONU sui diritti civili e politici del '66 (di seguito, anche Patto ONU). In particolare, il ricorrente evidenziava gli impatti diretti e mediati dell'innalzamento del livello del mare. Le più frequenti e regolari inondazioni (spesso tre o quattro volte al mese), 
distruggevano abitazioni e raccolti e provocavano una graduale erosione costiera e infiltrazioni saline, contaminando irrimediabilmente i pozzi di acqua dolce e rendendo il suolo povero e infertile. La compromissione dell'accesso all'acqua potabile, delle condizioni di salute e del ricorso ai mezzi di sussistenza andavano così a depauperare il diritto umano alla vita e a condurre una vita in maniera dignitosa. Nella sua decisione, il Comitato ONU anche se ritiene che la Nuova Zelanda, rimpatriando la famiglia Teitiota, non abbia violato il diritto alla vita (così come interpretato dal nuovo commento generale n. 36 del 2018), ha ritenuto astrattamente possibile che, in mancanza di robusti sforzi nazionali e internazionali, gli effetti del cambiamento climatico possano esporre gli individui alla violazione dei loro diritti ai sensi dell'art. 6 e 7 del Patto ONU (rispettivamente, diritto alla vita e divieto di trattamenti inumani o degradanti) e, di conseguenza, comportare il divieto di respingimento da parte dello Stato di rinvio (par. 9.11). Per comprendere la portata innovativa di tale principio deve osservarsi che per consolidata posizione del Comitato ONU, affinché vi fosse violazione del diritto umano alla vita ai sensi del Patto ONU era necessario provare che la minaccia fosse individuale, attuale o imminente. Con questa decisione, invece, la lesione del diritto umano alla vita viene per così dire anticipata e collocata nel momento in cui tale minaccia sia «reasonably foreseeble», cioè ragionevolmente prevedibile (par. 9.4). Inoltre, prosegue il Comitato, la circostanza che un intero Stato (il Kiribati) rischi di essere sommerso dall'innalzamento del livello del mare costituisce un rischio così estremo che le condizioni di vita possono diventare incompatibili col rispetto dell'art. 6 del Patto ONU, prima che tale evento sia imminente o che si realizzi (par. 9.11). La decisione, inoltre, vede il riconoscimento a livello internazionale del c.d. integrated approach nell'interpretazione dei diritti umani, in base al quale il depauperamento di diritti sociali come il diritto all'acqua e al cibo possono comportare la violazione di diritti di natura civile quale il diritto alla vita e il divieto di trattamenti inumani o degradanti. Infine, si assiste ad un ampliamento dell'ombrello protettivo del diritto alla vita che include, oltre alla minaccia di una lesione fisica o l'integrità mentale, anche il diritto degli individui a godere di una vita dignitosa e di essere liberi da atti o omissioni che causerebbero una morte innaturale o prematura (par. 9.4). Il divieto di respingimento di una persona verso un luogo dove vi sono fondati motivi per credere che vi sia un rischio reale di danno irreparabile al diritto umano alla vita e al divieto di trattamenti inumani o degradanti è un principio di diritto internazionale ben consolidato. La vera portata innovativa di questa decisione risiede nel riconoscimento di un collegamento tra eventi naturali e ambientali dovuti anche ai cambiamenti climatici e obblighi incombenti sugli Stati 
circa il rispetto dei diritti umani e, in particolare per quanto concerne l'oggetto del caso, il diritto alla vita.

\subsection{Riconoscimento della teoria degli obblighi positivi di tutela}

Il Comitato ONU, richiamando il par. 6 del nuovo commento generale n. 36 (2018), chiarisce che «deprivation of life involves a deliberate or otherwise foreseeable and preventable lifeterminating harm or injury, caused by an act or omission». La rilevanza della condotta omissiva segna il riconoscimento a livello internazionale della teoria degli obblighi positivi già emersa in ambito della giustizia regionale secondo cui, in chiave evolutiva, il contenuto normativo del diritto umano alla vita richiede agli Stati non solo l'obbligo negativo di astenersi dal creare un danno o una lesione intenzionale alla vita ma anche l'obbligo positivo di tutelare le «reasonably foreseeable threats and life-threatening situations that can result in loss of life» (par. 9.4).

In Teitiota viene effettuata una ricognizione delle più rilevanti pronunce rese dalla Corte Inter-americana dei diritti umani (Corte IADU) e della Corte europea dei diritti dell'Uomo (Corte EDU) in tema di protezione di diritti umani lesi da eventi naturali o ambientali dove viene riconosciuta la violazione del diritto alla vita e dell'integrità fisica in occasione di eventi ambientali di origine antropica o naturale (inclusi eventi improvvisi come un terremoto o un'inondazione) qualora, a seguito di un'opera di bilanciamento degli interessi, lo Stato non sia stato in grado di dimostrare di aver adottato le misure atte a salvaguardare le vite di coloro che erano sottoposti alla sua giurisdizione o non abbia adottato misure volte a ridurre al minimo gli effetti e i rischi di un inaspettato e violento fenomeno naturale. Nell'attribuire o meno la responsabilità ad uno Stato per la violazione di questi diritti non può chiedersi allo stesso un onere sproporzionato e impossibile ma è necessario prendere in considerazione un certo margine di apprezzamento nella scelta delle priorità (in base alle risorse disponibili) di ciascun Stato. In particolar modo, il giudizio sulla conformità della condotta statale all'obbligo positivo può dipendere da tre elementi: l'origine della minaccia, la chiara imminenza del rischio e la misura in cui questo poteva essere limitato, soprattutto nei casi di calamità che si abbattono sovente su una data area. È quindi richiesto a ciascun Stato parte l'obbligo primario di porre in essere un quadro legislativo e amministrativo volto a fornire appropriate deterrenze contro le minacce al diritto alla vita come l'obbligo di adottare pianificazioni territoriali e urbanistiche laddove necessario, l'obbligo di realizzare valutazioni 
di rischio ambientale, la creazione di regole obbligatorie per tutti quelli che sono implicati in attività rischiose volte a controllare la concessione delle licenze, la messa in opera, la sicurezza e la supervisione delle attività rischiose. Inoltre, incombe sullo Stato l'obbligo di informare le persone interessate di ogni emergenza possibile e dei potenziali rischi ambientali derivanti da fenomeni o attività umane o naturali che potrebbero influenzare il diritto alla vita (Corte EDU: Özel, 2015, parr.170-173; Kolyadenko, 2012, par.151; Budayeva, 2008, parr.129-132; Öneryildiz, 2004, parr. 89-90; Corte IADU, Advisory opinion, 2017, par. 47). In tal senso, la decisione in commento, inserendosi nel solco di queste pronunce, stabilisce il principio di diritto internazionale secondo cui la ragionevole prevedibilità di un evento naturale che possa minacciare il diritto alla vita o il godimento della stessa in maniera dignitosa, associata all'incapacità dello Stato di appartenenza di adempiere agli obblighi positivi di tutela, genera, in capo agli Stati terzi, l'obbligo negativo di non respingimento verso il Paese in questione.

Le conclusioni del Comitato ONU sono state criticate da alcuni dei relatori per aver richiesto un «unreasonable burden of proof» in capo al ricorrente e una «too high and unreasonable threshold» richieste dal Comitato ONU (Annex 2, par. 3). Per comprendere tale ragionamento è necessario considerare che il Patto ONU, come, in generale, gli altri strumenti internazionali a presidio della tutela dei diritti umani, è stato storicamente creato per proteggere gli individui dalla violazione di diritti in contesti di man-made disasters (violenze individuali, conflitti, disordini pubblici, torture etc.) mentre, nella tradizione giuridica internazionale, era (fino a poco tempo fa) del tutto nuovo pensare di imputare allo Stato di appartenenza la responsabilità per la violazione dei diritti umani a seguito di eventi naturali o ambientali (indotti anche dal cambiamento climatico). Ciò è ben espresso dalla lettura congiunta della decisione in commento con la richiamata sentenza della Corte EDU resa in Sufi and Elmi (2011) con cui si chiarisce che la soglia di gravità (threshold) della lesione richiesta per creare un rischio reale di un danno irreparabile ai sensi degli artt. 6 e 7 del Patto ONU deve essere estremamente alta in quanto in questi casi il pregiudizio futuro ipotizzato non proverrebbe da atti o omissioni intenzionali ma da una condizione generale (nel caso di specie il cambiamento climatico) (par. 9.7). Tuttavia, nell'evoluzione giurisprudenziale, a parità del bene giuridico tutelato, le cause e le circostanze in cui questo rischia di essere minacciato incidono sulla soglia di lesione richiesta per potersi configurare violazione del diritto stesso. Ad esempio, qualora si accertasse che l'individuo è fuggito oltre i confini del proprio Stato a causa di un determinato evento naturale o ambientale che è il risultato, non di una condizione generale (come nel caso al vaglio del Comitato), ma di una cosciente azione od omissione 
umana (quale ad esempio le pratiche di land and water grabbing) la threshold richiesta sarebbe meno stringente (HRC Portillo Cáceres et al., 2016, par. 7.4; Corte EDU, M.S.S., 2011, ACHPR, Serac, 1996, par. 44)

\section{Considerazioni finali: migrazioni internazionali indotte da motivi ambientali e applicazione della dichiarazione di cartagena sui rifugiati?}

Se da un lato il Comitato Onu rende la sua decisione in un'ottica di diritto internazionale dei diritti umani e in alcun modo entra nel merito dei criteri per la qualifica di rifugiato, dall'altro, il membro del Comitato ONU Yuval Shany questo precedente «sets forth new standards that could facilitate the success of future climate change-related asylum claims». Muovendosi quindi dal lato giurisprudenziale a quello legislativo è interessante analizzare in che misura $\mathrm{i}$ principi di diritto internazionale emersi in questa pronuncia possano incidere in vista di una possibile applicazione della definizione regionale di rifugiato prevista dalla Dichiarazione di Cartagena del 1948 nei confronti delle persone che sono fuggite dal Paese di origine per motivi ambientali. La Dichiarazione di Cartagena offre protezione a coloro che «han huido de sus países porque su vida, seguridad o libertad han sido amenazadas por la violencia generalizada, la agresión extranjera, los conflictos internos, la violación masiva de los derechos humanos $u$ otras circunstancias que hayan perturbado gravemente el orden público».

Una delle principali difficoltà nel ritenere applicabile la definizione regionale di rifugiato ai migranti ambientali risiede nel considerare gli eventi violaciones masivas de derechos humanos e otras circunstancias que hayan perturbado gravemente el orden público come il risultato di condotte umane e che non facesse riferimento ai disastri ambientali (CIREFCA, 1989, parr. 33 e 38 e UNHCR' 1992, par. 40). In estrema sintesi questa posizione riflette il c.d hazard paradigm che concettualizzava le persone che rischiano l'esposizione ai danni causati dagli eventi naturali e ambientali come vittime della forza indiscriminata della natura e dove l'incidenza dell'attività umana è pressoché assente (SCOTT, 2016, p 32 e SCOTT, 2020, p. 12). Successivamente, nell'ultimo decennio sono stati resi documenti e pareri interpretativi di soft law tesi ad estendere l'ombrello protettivo della Dichiarazione di Cartagena nei confronti

\footnotetext{
${ }^{1}$ La Corte africana, nel ritenere il governo nigeriano responsabile per la violazione del diritto alla vita ai danni degli Ogoni ritiene che «The pollution and environmental degradation to a level humanly unacceptable has made it living in the Ogoni land a nightmare [...] These and similar brutalities not only persecuted individuals in Ogoniland but also the whole of the Ogoni community as a whole. They affected the life of the Ogoni society as a whole» 
delle migrazioni transfrontaliere indotte da motivi ambientali (CANTOR, 2015 e PLAN DE ACCIÓN DE BRASIL, 2014, p. 19). Ad esempio, in vista della commemorazione del trentennale della Dichiarazione, Cartagena +30, sotto l'egida dell'UNHCR è stata organizzata una tavola rotonda di esperti chiamati a delineare l'attuale e più recente evoluzione interpretativa della Dichiarazione. Nel documento prodotto, Resumen de las conclusiones sobre la interpretación de la definición ampliada de refugiado de la Declaración de Cartagena de 1984, alcuni esperti evidenziavano come la definizione di rifugiato regionale dovesse essere intesa in maniera flessibile tale da concedere agli Stati una certa autonomia nel decidere se garantire la protezione in quei casi non ricoperti dalle altre situazioni riportate in convenzione, come il caso di disastri naturali (2013, par. 26). Successivamente, il parere dell'Alto Commissariato delle Nazioni Unite per i Rifugiati, Climate change, disaster and displacement in the Global Compacts: UNHCR's perspectives ritiene che «People fleeing extreme situations of disasters and adverse effects of climate change indeed may be compelled to leave their country of origin because of events seriously disturbing public order». (2017, p. 2).

La decisione del Comitato ONU che abbiamo analizzato segna indubbiamente, un punto di svolta epocale in materia di tutela dei diritti umani. Difatti seppur non sia giuridicamente vincolante, il Patto ONU sui diritti civili e politici e la giurisprudenza a cui fa riferimento lo sono. Il Comitato segnando che il riconoscimento che l'impatto degli eventi ambientali e naturali possano sollevare una seria minaccia all'abilità di godere del diritto alla vita e del divieto di trattamenti inumani o degradanti e che l'incapacità dello Stato di appartenenza di adempiere agli obblighi positivi di tutela, genera, in capo agli Stati terzi, l'obbligo negativo di non di respingimento verso il Paese in questione, ha il potenziale di aprire le porte al riconoscimento della categoria dei rifugiati per motivi indotti da eventi naturali o ambientali.

\section{Referencias}

SCOTT, M., Climate Change, disasters, and the Refugee Convention, Cambridge, Cambridge University Press, 2020.

INTERNAL MONITORING CENTER, Global Report on Internal Displacement 2020 https://www.internal-displacement.org/, asseso em 22 jul 2020.

UNITED NATIONS GENERAL ASSEMBLY, Providing legal protection for persons displaced by the impact of climate change, A/73/L.105, 2019. 
UNITED NATIONS, Report of the United nations High Commissioner for Refugees: Global Compact on Refugees, A/73/12 (Part II), 2018.

HUMAN RIGHTS COMMITTEE, General comment No. 36 (2018) on article 6 of the International Covenant on Civil and Political Rights, on the right to life, CCPR/C/GC/36, 2018 .

UN HIGH COMMISSIONER FOR REFUGEES, Climate change, disaster and displacement in the Global Compacts: UNHCR's perspectives, 2017.

SCOTT, M., Finding Agency in Adversity: Applying the Refugee Convention in the Context of Disasters and Climate Change, Refugee Survey Quarterly, v. 35, n. 4, p. 26-57, 2016.

CANTOR, D., Leyes, Polìticas y pràcticas en materia de protecciòn temporale humanitaria de extranjeros en el contexto de desastres, San José, Costa Rica, Nansen Initiative, 10-11 de febrero 2015.

PLAN DE ACCIÓN DE BRASIL, Una hoja de ruta común para fortalecer la protección y promover soluciones sostenibles para las personas refugiadas, desplazadas y apátridas en América Latina y el Caribe dentro de un Marco de cooperación y Solidaridad, Brasilia, 3 de diciembre de 2014.

UN HIGH COMMISSIONER FOR REFUGEES, Reunión de expertos Interpretación de la definición ampliada de refugiado contenida en la Declaración de Cartagena sobre Refugiados de 1984, Montevideo, 15 y 16 de octubre de 2013.

Antropocene, Lessico del XXI Secolo, Treccani, 2012.

UN HIGH COMMISSIONER FOR REFUGEES, Persons covered by the Oua Convention Governing the Specific Aspects of Refugees Problems in Africa and by the Cartagena Declaration on Refugees (Submitted by the African Group and the Latin American Group), EC/1992/SC, 1-17, 6 April 1992.

CONFERENCIA INTERNACIONAL SOBRE REFUGIADOS CENTROAMERICANOS, Principios y criterios para la proteccion y asistencia a los refugiados, repatriados y desplazados centroamericanos en america latina, Ciudad de Guatemala, CIREFCA 89/9 Abril 1989.

\section{Giurisprudenza}

UN HUMAN RIGHTS COMMITTEE, Ioane Teitiota v. New Zealand (advance unedited version), 7 January 2020, CCPR/C/127/D/2728/2016.

INTER-AMERICAN COURT OF HUMAN RIGHTS, Advisory opinion OC-23/17 of 15 November 2017 on the environment and human rights, series A, No. 23.

INTER-AMERICAN COURT OF HUMAN RIGHTS Portillo Cáceres et al. v. Paraguay (CCPR/C/126/D/2751/2016). 
CORTE EUROPEA DEI DIRITTI DELL'UOMO M. Özel and others v. Turkey ,17 November 2015, 14350/05, 15245/05 and 16051/05.

CORTE EUROPEA DEI DIRITTI DELL'UOMO, MSS c. Belgio e Grecia, 21 gennaio 2011 [GC], 30696/09.

CORTE EUROPEA DEI DIRITTI DELL'UOMO Budayeva and others v. Russia, 20 March 2008, 15339/02, 21166/02, 20058/02, 11673/02 and 15343/02.

CORTE EUROPEA DEI DIRITTI DELL'UOMO, Öneryildiz v. Turkey, 30 November 2004 [GC], 48939/99.

AFRICAN COURT ON HUMAN AND PEOPLES' RIGHTS, Communication 155/96 Social and Economic Rights Action Center (SERAC) and Center for Economic and Social Rights (CESR) / Nigeria, 27 October 2001. 